\title{
Video-assisted thoracoscopic surgery in lung cancer staging
}

\author{
Frederico Krieger Martins, Guilherme Augusto Oliveira, Juliano Cé Coelho, Márcio Chmelnitsky \\ Kruter, Thiago Krieger Bento da Silva, Juliana Scheffer, Marcelo Bastiani Pasa
}

Núcleo de Oncologia Torácica, Hospital do Câncer Mãe de Deus, Porto Alegre, Brazil

Contributions: (I) Conception and design: FK Martins, GA Oliveira, JC Coelho; (II) Administrative support: TK Bento da Silva, MC Kruter, MB Pasa; (III) Provision of study materials or patients: All authors; (IV) Collection and assembly of data: All authors; (V) Data analysis and interpretation: All authors; (VI) Manuscript writing: All authors; (VII) Final approval of manuscript: All authors.

Correspondence to: Frederico Martins. Núcleo de Oncologia Torácica, Hospital do Câncer Mãe de Deus, Rua Costa, 40, CEP 90110-270, Porto Alegre, Brazil. Email: fredericokm@gmail.com.

\begin{abstract}
Video-assisted thoracoscopic surgery (VATS) is a minimally invasive technique enabling a much more comfortable postoperative recovery. It can be a very useful approach in staging lung cancer addressing all components of TNM classification and, so, indicate the best therapeutic option in each case. In this article we describe the contribution of VATS in each of the components (T, N, M) and present some examples of our center.
\end{abstract}

Keywords: Staging; lung cancer; endobronchial ultrasound-endoscopic ultrasound (EBUS-EUS); mediastinoscopy; video-assisted thoracoscopic surgery (VATS)

Received: 31 July 2018; Accepted: 09 October 2018. Published: 06 November 2018.

doi: 10.21037 /jovs.2018.10.02

View this article at: http://dx.doi.org/10.21037/jovs.2018.10.02

\section{Introduction}

Lung cancer represents a health issue worldwide, specially in Brazil. Once its incidence started to decline after the campaigns against tobacco, it is still by far the leading cause of mortality. The 5-year overall survival (OS) for the entire population remains low and the appropriate disease staging has direct implication in the treatment and in the disease prognosis. For clinical staging, guidelines recommend the use of PET-CT (1), although imaging work up with computed tomography (CT) or nuclear magnetic resonance (NMR) are appropriate in the absence of PET-CT. Once the images methods have only good accuracy the pathologic staging can differ from the clinical staging in up to a third of lung cancer cases.

Surgery presents the best treatment options for early stage disease. Therefore, an appropriate patient selection is critical to arrises the best results. Defining the most advanced component in the TNM classification is very important for understanding the extent of disease. Despite major advances in the two last decades, sometimes imaging tests may fail and either under or over-stage the disease (2). In this way, VATS may be a good option as it is a minimally invasive approach, reducing injury and complications inherent to a standard thoracotomy (3).

This article reviews the role of VATS in each component of the TNM in lung cancer from the perspective of a Brazilian thoracic surgery team.

\section{Assessing the T status}

The $T$ status defines the tumor size and its relations with other pulmonary and mediastinal structures. Images technique usually can well define the $\mathrm{T}$ component, but it could by specially difficult in some particular situation. Tumors invading chest wall (T3) result in higher postoperative morbidity as well as worse prognosis. Usually, the degree of invasion is well defined preoperatively through computed tomography (CT) or magnetic resonance imaging (MRI) of the thorax. However, there are situations where the signs are not so obvious. For instance, exclusive invasion of the parietal pleura is revealed, sometimes, only in the intraoperative period, since its radiological findings are frequently tenuous. In this situation, VATS reveals the need for an extrapleural resection and also assists in obtaining adequate surgical margins. When there is a gross 
invasion of the chest wall, VATS may be helpful by guiding the approach and defining the limits of a complete en bloc removal.

Mostly tumors classified as T4 are not eligible for surgical treatment because they affect vital structures such as the heart, large vessels, and trachea. Despite this, there is a subgroup of patients who benefits from resection. This should always be a multidisciplinary decision. The improvement of surgical techniques and equipments have made VATS technique feasible and safe in selected cases of T4 tumors in experienced hands. VATS may also anticipate the need to convert into open surgery cases with complex anatomy due to tumor invasion. Finally, it can also interrupt the progress of the planned procedure avoiding futile measures.

Patients with a suspicious ipsilateral lesion in a different lobe can also be classified as T4 according to individualization of the case. VATS wedge resection of the accessible lesion can provide diagnosis and consequently guide the need for further adequate resection.

\section{Assessing the $\mathbf{N}$ status}

Lymph node involvement is the major determinant of prognosis and treatment in non-small cell lung cancer (NSCLC). Thus, proper evaluation of the $\mathrm{N}$ status is critical.

After initial investigation, some individuals can be submitted to surgical treatment promptly. On the other hand, patients with suspected N1 nodes, central tumors and peripheral tumors larger than $3 \mathrm{~cm}$ need invasive mediastinal staging even in the setting of normal CT or PET-CT. Combined endobronchial ultrasound (EBUS)endoscopic ultrasound (EUS), mediastinoscopy and VATS are the most common procedures for this purpose (4-6).

EBUS-EUS is currently the gold standard for nodal staging when performed by an experienced operator. However, this is not yet a widely available method.

VATS exposes the entire pleural cavity, enabling almost all ipsilateral nodal stations to be analyzed. This way N2 lymph nodes are easily reached. However, N3 (contralateral) lymph nodes are not anatomically accessible. This is a major limitation of VATS.

In this context, mediastinoscopy persists as the most widespread technique nowadays, since it allows access to the N2 and N3 lymph nodes.

Despite the above, VATS plays an important role in mediastinal staging in the following situations:

* Sublobar resections;

* Hostile mediastinum;

* Approach to aorto-pulmonary nodal zone.

There is much discussion about sublobar resections. Saving parenchyma is of paramount importance in patients with limited pulmonary function. However, this can not be detrimental to the criteria of adequate oncological resection. The two main ongoing clinical trials on the subject (CALGB140503 and JCOG0802/WJOG4607) use $\mathrm{N} 0$ as an eligibility criteria. Lesions with $\mathrm{N} 1$ involvement are classified as locally advanced disease (7). Thus, it would be appropriate to guarantee the absence of $\mathrm{N} 1$ involvement before indicating a sublobar resection to preserve pulmonary parenchyma.

Hostile mediastinum may be called any situation in which mediastinoscopy is difficult to perform. For instance, in the case of a previous mediastinoscopy, tracheoplasty or anatomical cervical defect. VATS is an alternative access to be considered.

Tumors located on the left side, mainly in the upper lobe, can metastasize to stations 5 and 6 (aortopulmonary zone) (8). EBUS-EUS can reach these lymph nodes, but this is an advanced technique with good performance only in experienced centers. VATS approach enables easy and secure access to these stations.

\section{Assessing the M status}

Individuals with pleural or pericardial implants are classified as stage IVA. Therefore, these patients are not eligible for surgical resections. Instead, they will receive systemic therapy generally with palliative intent. Thus, careful inspection of pleural cavity is of paramount importance during VATS.

Daily, thoracic surgeons manage pleural effusions with a high risk of malignancy. In this scenario, VATS is either diagnostic, providing adequate tissue for analysis, and therapeutic, enabling pleurodesis or partial pericardiectomy for symptoms relief.

Sometimes pleural spread is an unexpected intraoperative finding. In this case, the planned procedure is altered avoiding a futile pulmonary resection or even a futile thoracotomy.

Patients with lung cancer may have more than one lesion at the presentation or develop a new one over time. In cases where there is a contralateral lesion, the approach 

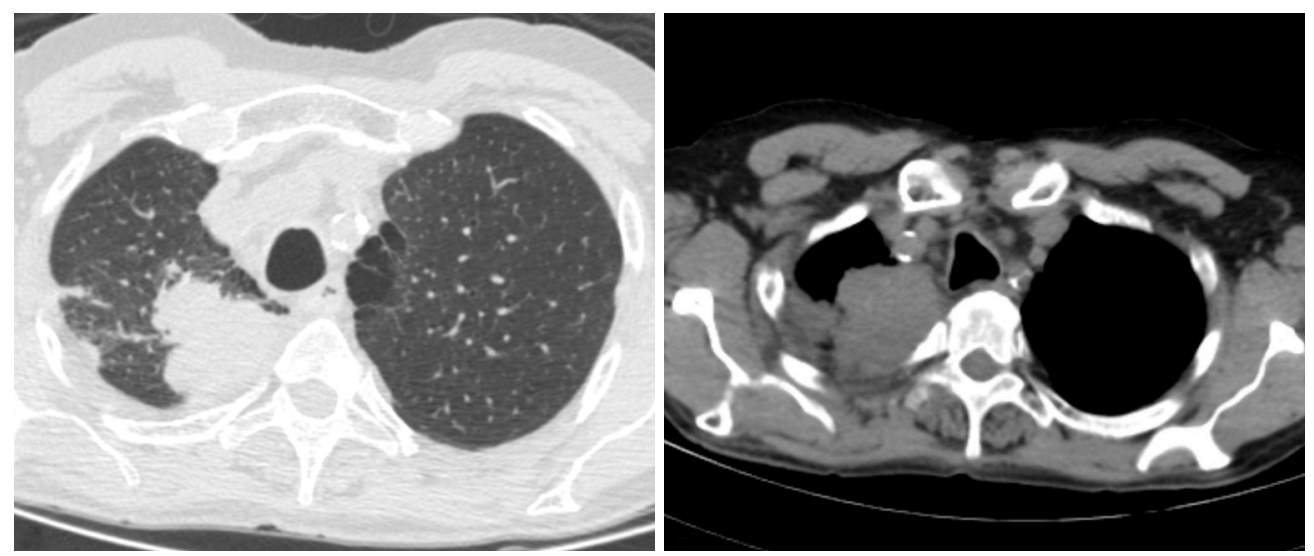

Figure 1 Right upper lobe mass invading chest wall.

through VATS may aid in the differentiation between metastasis or primary lung cancer, which will result in different treatments.

\section{Our practice}

We routinely initiate any curative resection by VATS, regardless of whether the planned surgical approach is minimally invasive or open.

Our preferred access is two-port technique. The camera port $(10 \mathrm{~mm})$ is placed at 8 th intercostal space in the anterior axillary line. The second port $(5 \mathrm{~mm})$ is inserted in the planned line of utility incision or thoracotomy. In this way we can inspect the parietal pleural. Full incision is done only if no implants are found.

Through a $5 \mathrm{~cm}$ long incision, usually at 5 th intercostal space in the axillary line, it is possible to mobilize lung posteriorly and evaluate hilar anatomy. According the hilum involvement, the need for a thoracotomy can be anticipated or the planned procedure discontinued.

Patients supposedly with early disease, after careful inspection of pleura and hilum we proceed with systematic lymph node sampling. On the right side lesions, stations 2R, 4R, 7 and $10 \mathrm{R}$ are sampled and, on the left, 5, 6, 7, and $10 \mathrm{~L}(9)$. These lymph nodes are submitted to frozen section (10). If the analysis is negative we complete the proposed lung resection. However, in the case of lymph node involvement a mediastinal lymph node dissection is indicated.

Individuals with more advanced lesions but still fulfilling criteria for surgical treatment should go straight to mediastinal lymph node dissection.

\section{Some of our cases}

\section{Case 1}

A 57-year-old man presenting with a large right upper lobe non-small cells lung cancer invading chest wall (T3N0M0IIB) (Figure 1). After induction chemoradiotherapy a radical resection were performed. A posterolateral (ShawPaulson) approach was planned. Anyway, the surgery started by VATS to guide the thoracotomy, surgical margins and exclude pleural implants. After this, a right upper lobectomy with chest wall en bloc resection and mediastinal lymph node dissection were performed (Figure 2).

\section{Case 2}

A 57-year-old woman with a left upper lobe adenocarcinoma (Figure 3). In the intraoperative period it was not possible to mobilize lung posteriorly. Through this we confirmed the involvement of the pericardium (T3N0M0-IIB). The opening of the pericardium revealed that a complete resection would be possible. A VATS left upper lobectomy with pericardium en bloc resection and lymph node dissection were performed.

\section{Case 3}

Centrally located lung lesion in a 77-year-old woman (Figure 4). Bronchoscopy made the diagnosis of adenocarcinoma and revealed a safe surgical margin for a left lower lobectomy. However, it was not possible through the CT to rule out involvement of great vessels or even left atrium. VATS has excluded extensive hilar involvement, 

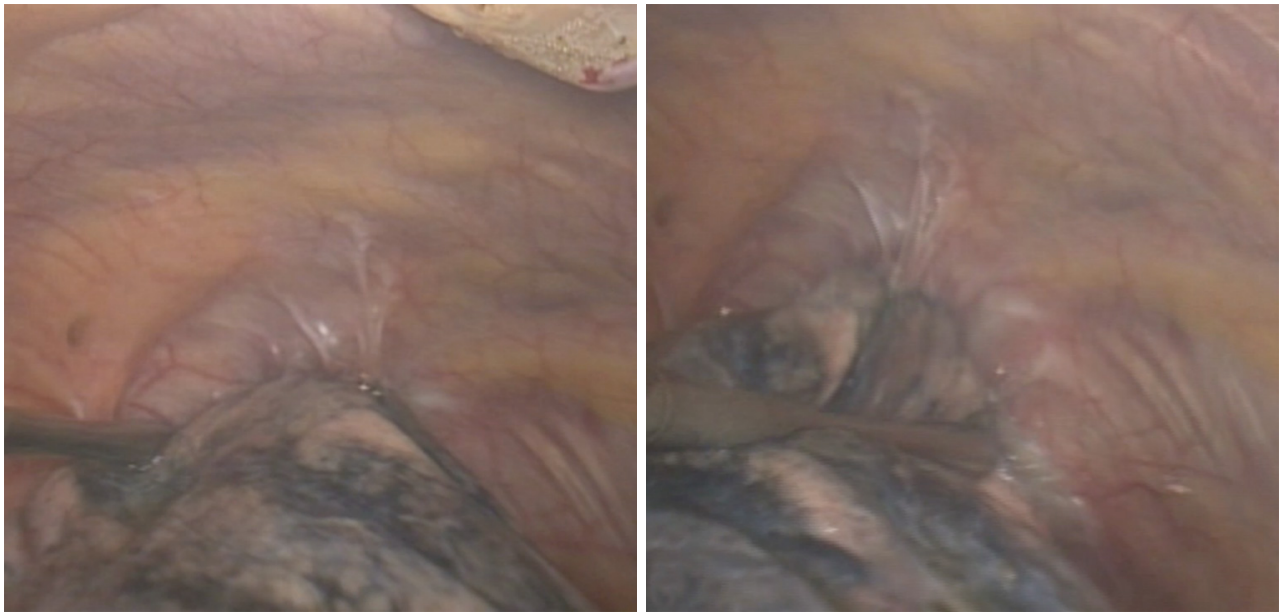

Figure 2 VATS pleural inspection demonstrating the need of en bloc resection. VATS, video-assisted thoracoscopic surgery.
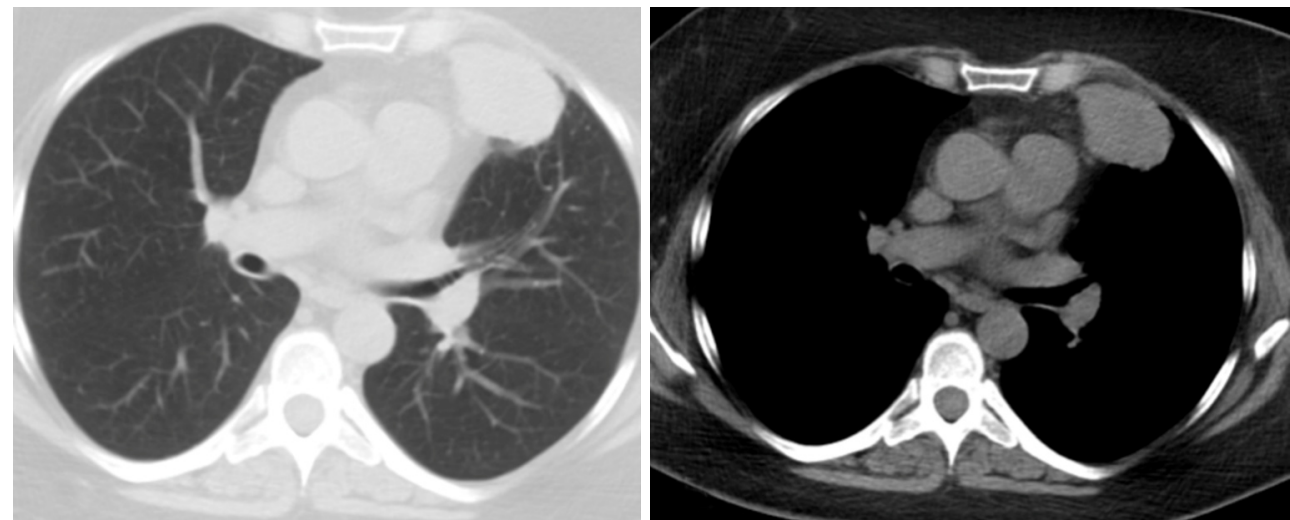

Figure 3 Left upper lobe adenocarcinoma with pericardium involvement.
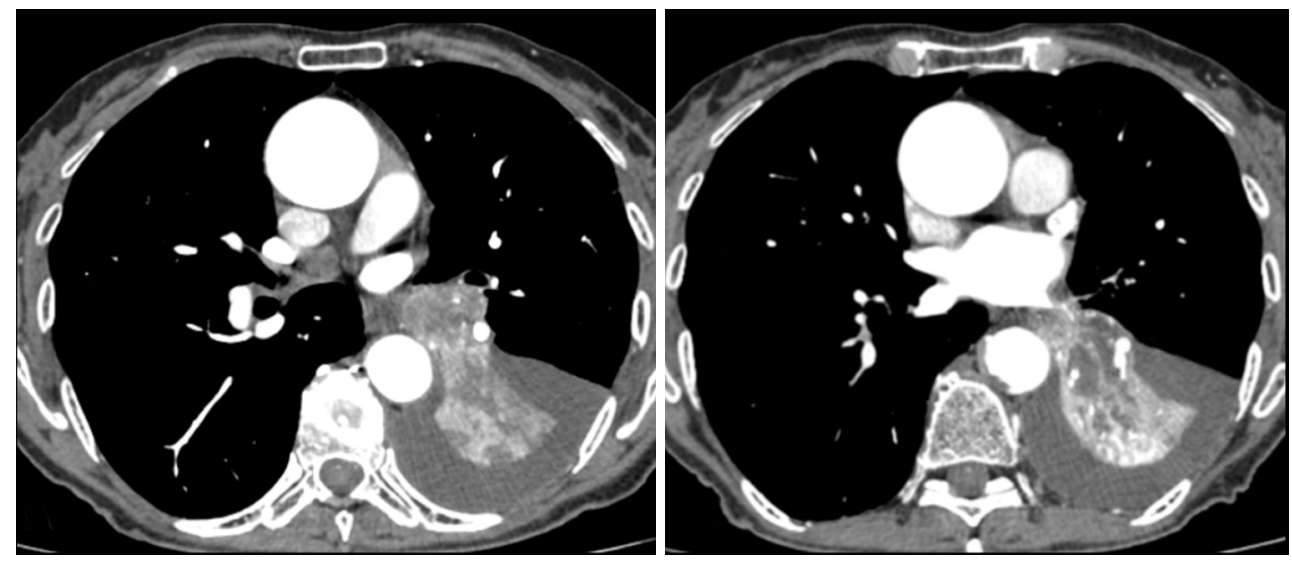

Figure 4 CT showing a centrally located NSCLC near the left atrium. NSCLC, non-small cell lung cancer. 
but demonstrated that the lesion was resectable only by intrapericardial pneumonectomy (T3N0M0-IIB). At this point, we decided convert to conventional open surgery (Figure 5).

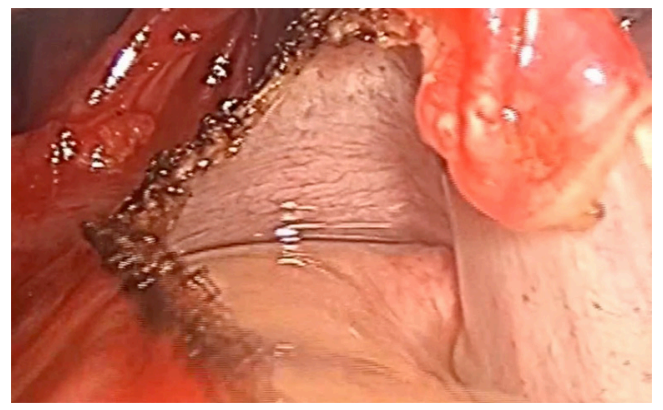

Figure 5 VATS pericardial opening revealing adequate surgical margin por intrapericardial pneumonectomy. VATS, video-assisted thoracoscopic surgery.

\section{Case 4}

A 63-year-old woman with synchronous ipsilateral lesions (middle and right lower lobes) (Figure 6). CT appearance suggested two separate primary tumors. Mediastinoscopy without lymph node involvement. At the surgery, systematic lymph node sampling with frozen section again excluded malignancy. Bilobectomy (middle and right lower lobes) specimen revealed multiple primary lung cancer. Middle lobe (T2aN0M0-IB). Right lower lobe (T1aN0M0-IA1).

\section{Case 5}

Metachronous left upper lobe lung lesion in a 59-yearold woman with previous right lower lobectomy to treat a lung adenocarcinoma 3 years before (T3N2M0-IIIB) (Figure 7). PET-CT has not demonstrated any other hypermetabolic process besides the lung lesion. Patient had already undergone mediastinoscopy in the time of
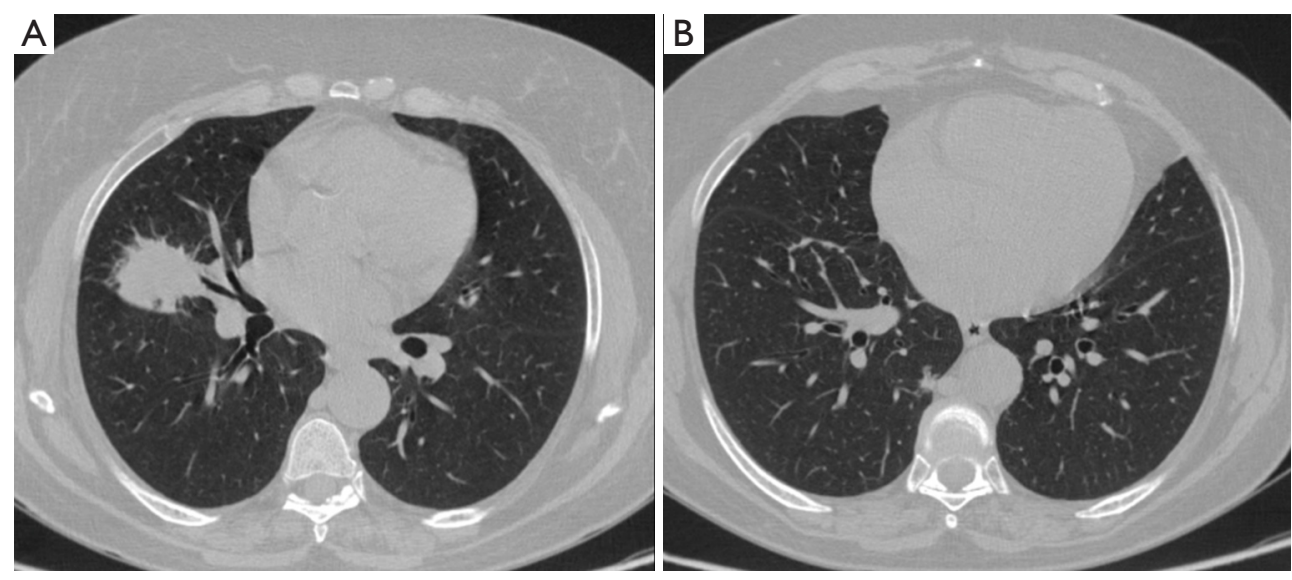

Figure 6 Synchronous ipsilateral lesions. (A) middle lobe; (B) right lower lobe.
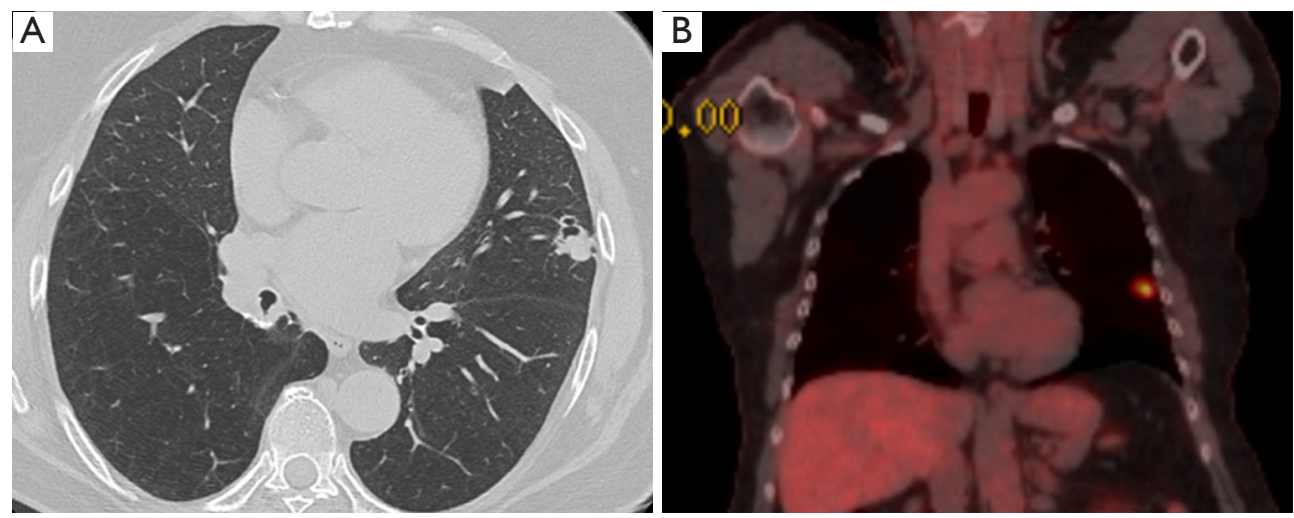

Figure 7 Left upper lobe lesion. (A) CT; (B) PET-CT. 
first malignancy. Therefore, the resection started by systematic lymph node sampling with intraoperative frozen section. The negative result allowed us to proceed with VATS lingulectomy. The final diagnosis was a new primary lung adenocarcinoma (T1cN0M0-IA3) (Figure 8).

\section{Case 6}

Patient with right upper lobe nodule highly suggestive of neoplasia, but without diagnosis at the time of surgery (Figure 9). Intraoperative inspection of the parietal pleura showed a small and unexpected lesion. Frozen section excluded malignant pleural implant. Systematic lymph node sampling with frozen section and consequently lobar resection were completed. The nodule was an adenocarcinoma (T1bN0M0-IA2).

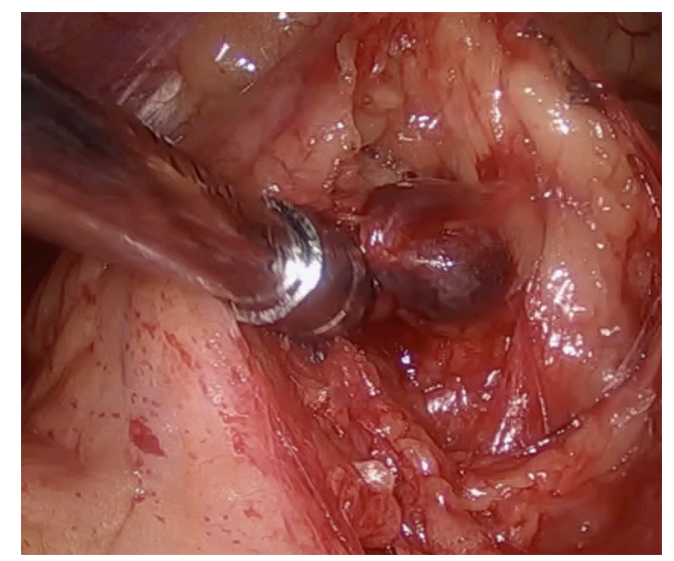

Figure 8 Aorto-pulmonary nodal zone during VATS systematic lymph node sampling. VATS, video-assisted thoracoscopic surgery.

\section{Conclusions}

VATS has been prominent in the treatment of lung cancer through the evolution of the lobectomy technique. Nevertheless, it can be a very useful approach to staging.

VATS can be a useful approach in each one of the TNM components. This is particularly true in mediastinal lymph nodes evaluation, especially in centers where EBUS-EUS is not easily available, as is the case in Brazil.

\section{Acknowledgments}

Funding: None.

\section{Footnote}

Provenance and Peer Review: This article was commissioned by the Guest Editors (Ricardo M. Terra and Paula A. Ugalde) for the series "Minimally Invasive Surgery Robotics and VATS in Brazil" published in Fournal of Visualized Surgery. The article has undergone external peer review.

Conflicts of Interest: All authors have completed the ICMJE uniform disclosure form (available at http://dx.doi. org/10.21037/jovs.2018.10.02). The series "Minimally Invasive Surgery - Robotics and VATS in Brazil" was commissioned by the editorial office without any funding or sponsorship. The authors have no other conflicts of interest to declare.

Ethical Statement: The authors are accountable for all aspects of the work in ensuring that questions related to the accuracy or integrity of any part of the work are
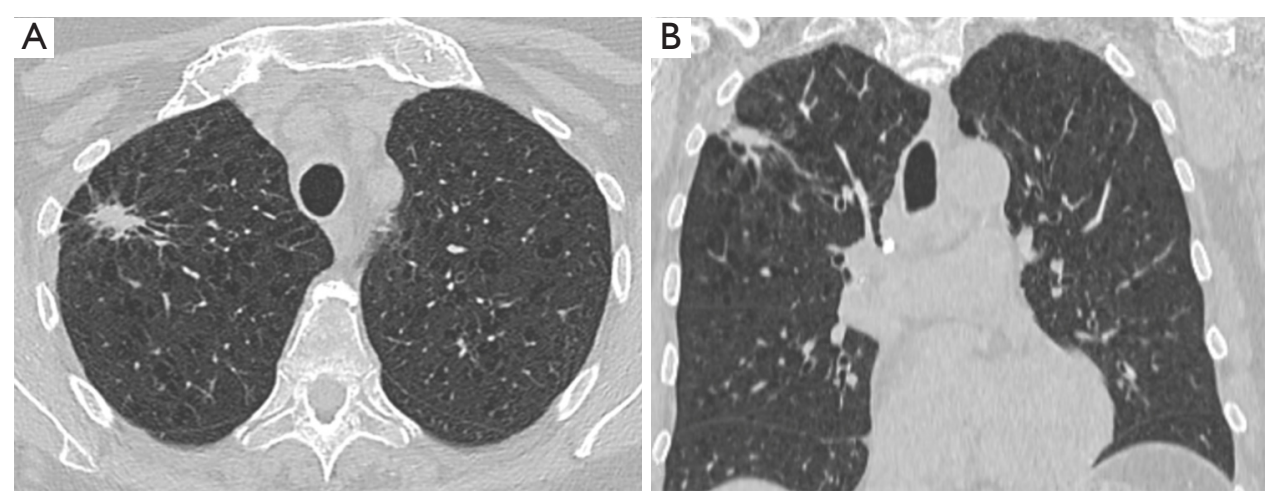

Figure 9 CT with right upper lobe lesion. (A) axial plane; (B) sagittal plane. 
appropriately investigated and resolved.

Open Access Statement: This is an Open Access article distributed in accordance with the Creative Commons Attribution-NonCommercial-NoDerivs 4.0 International License (CC BY-NC-ND 4.0), which permits the noncommercial replication and distribution of the article with the strict proviso that no changes or edits are made and the original work is properly cited (including links to both the formal publication through the relevant DOI and the license). See: https://creativecommons.org/licenses/by-nc-nd/4.0/.

\section{References}

1. Fischer B, Lassen U, Mortensen J, et al. Preoperative staging of lung cancer with combined PET-CT. N Engl J Med 2009;361:32-9.

2. Saettele TM, Ost DE. Multimodality systematic approach to mediastinal lymph node staging in non-small cell lung cancer. Respirology 2014;19:800-8.

3. Yim AP. Routine video-assisted thoracoscopy prior to thoracotomy. Chest 1996;109:1099-100.

4. Detterbeck FC, Jantz MA, Wallace M, et al. Invasive mediastinal staging of lung cancer: ACCP evidencebased clinical practice guidelines ( $2 \mathrm{nd}$ edition). Chest 2007;132:202S-20S.

doi: 10.21037 /jovs.2018.10.02

Cite this article as: Martins FK, Oliveira GA, Coelho JC, Kruter MC, da Silva TK, Scheffer J, Pasa MB. Video-assisted thoracoscopic surgery in lung cancer staging. J Vis Surg 2018;4:230.
5. De Leyn P, Dooms C, Kuzdzal J, et al. Revised ESTS guidelines for preoperative mediastinal lymph node staging for non-small-cell lung cancer. Eur J Cardiothorac Surg 2014;45:787-98.

6. Hegde PV, Liberman M. Mediastinal staging: endosonographic ultrasound lymph node biopsy or mediastinoscopy. Thorac Surg Clin 2016;26:243-9.

7. Sesti J, Donington JS. Sublobar resection: ongoing controversy for treatment for stage I non-small cell lung cancer. Thorac Surg Clin 2016;26:251-9.

8. Cerfolio RJ, Bryant AS, Eloubeidi MA. Accessing the aortopulmonary window (\#5) and the paraaortic (\#6) lymph nodes in patients with non-small cell lung cancer. Ann Thorac Surg 2007;84:940-5.

9. Darling GE, Allen MS, Decker PA, et al. Randomized trial of mediastinal lymph node sampling versus complete lymphadenectomy during pulmonary resection in the patient with N0 or N1 (less than hilar) non-small cell carcinoma: results of the American College of Surgery Oncology Group Z0030 Trial. J Thorac Cardiovasc Surg 2011;141:662-70.

10. Li W, Yang XN, Liao RQ, et al. Intraoperative frozen sections of the regional lymph nodes contribute to surgical decision-making in non-small cell lung cancer patients. J Thorac Dis 2016;8:1974-80. 